\title{
Langmuir
}

This document is confidential and is proprietary to the American Chemical Society and its authors. Do not copy or disclose without written permission. If you have received this item in error, notify the sender and delete all copies.

\section{Nanoplasmonic Sensing at the Carbon-Bio Interface: Study of Protein Adsorption at Graphitic and Hydrogenated Carbon Surfaces}

\begin{tabular}{|r|l|}
\hline Journal: & Langmuir \\
\hline Manuscript ID & la-2017-00612n.R1 \\
\hline Manuscript Type: & Article \\
\hline Date Submitted by the Author: & $\mathrm{n} / \mathrm{a}$ \\
\hline Complete List of Authors: & $\begin{array}{l}\text { Zen, Federico; Trinity College Dublin, School of Chemistry } \\
\text { Karanikolas, Vasilios; University of Dublin Trinity College, Physics } \\
\text { Behan, James; Trinity College Dublin, School of Chemistry } \\
\text { Andersson, Jenny; Insplorion AB } \\
\text { Ciapetti, Guido; Trinity College Dublin, School of Chemistry } \\
\text { Bradley, A. Louise; Trinity College Dublin, School of Physics } \\
\text { Colavita, Paula; Trinity College Dublin, School of Chemistry }\end{array}$ \\
\hline \hline
\end{tabular}

SCHOLARONE

Manuscripts 


\title{
Nanoplasmonic Sensing at the Carbon-Bio Interface:
}

\section{Study of Protein Adsorption at Graphitic and}

\section{Hydrogenated Carbon Surfaces}

\author{
Federico Zen, ${ }^{a}$ Vasilios D. Karanikolas, ${ }^{b}$ James A. Behan, ${ }^{a}$ Jenny Andersson, ${ }^{c}$ Guido Ciapetti, ${ }^{a}$ \\ A. Louise Bradley, ${ }^{b}$ Paula E. Colavita ${ }^{a^{*}}$ \\ a - School of Chemistry and AMBER Research Centre, College Green, Trinity College Dublin, \\ Ireland. \\ b - School of Physics and CRANN, College Green, Trinity College Dublin, Ireland. \\ c - Insplorion AB, Medicinaregatan 8A, 41390 Göteborg, Sweden
}




\section{Abstract}

Various forms of carbon are known to perform well as biomaterials in a variety of applications and an improved understanding of their interactions with biomolecules, cells and tissues is of interest for improving and tailoring their performance. Nanoplasmonic sensing (NPS) has emerged as a powerful technique for studying the thermodynamics and kinetics of interfacial reactions. In this work, the in situ adsorption of two proteins, bovine serum albumin and fibrinogen, were studied at carbon surfaces with differing chemical and optical properties using nanoplasmonic sensors. The carbon material was deposited as a thin film onto NPS surfaces consisting of $100 \mathrm{~nm} \mathrm{Au}$ nanodisks with a localized plasmon absorption peak in the visible region. Carbon films were fully characterized by x-ray photoelectron spectroscopy (XPS), atomic force microscopy (AFM) and spectroscopic ellipsometry (SE). Two types of material were investigated: amorphous carbon (a-C) with high graphitic content and high optical absorptivity, and hydrogenated amorphous carbon (a-C:H), with low graphitic content and high optical transparency. The optical response of the Au/carbon NPS elements was modelled using the finite difference time domain (FDTD) method, yielding simulated analytical sensitivities that compare well with those observed experimentally at the two carbon surfaces. Protein adsorption was investigated on a-C and a-C:H and the protein layer thicknesses were obtained from FDTD simulations of the expected response, yielding values in the 1.8-3.3 nm range. A comparison of the results at a-C and a-C:H indicates that in both cases fibrinogen layers are thicker than those formed by albumin by up to $80 \%$. 


\section{Introduction}

Carbon coatings, such as amorphous carbon (a-C) and hydrogen-doped carbon (a-C:H), have emerged as good biomaterials and been integrated into several biodevices like catheters, stents, sensors, medical guidewires, surgical needles, orthopedic implants and prostheses. ${ }^{1-3}$ The success of carbon coatings in biological applications is partly due to a combination of physical/chemical properties that underpins their good performance, such as chemical inertness, low frictional coefficient and high wear resistance. ${ }^{4-5}$ However, the durability, functionality and bioresponse of artificial materials in vivo are limited by their interaction with blood and tissue ${ }^{2,6}$. The competitive adsorption of plasma proteins (such as albumin and fibrinogen), occurring at an early stage after implantation, is considered to have a crucial effect in determining the response of the host when in contact with biomaterials. ${ }^{6-10}$ For this reason, much effort has been dedicated to the description of protein adsorption and/or binding at carbon surfaces with different physical and chemical properties, by using both in situ and ex situ methods. ${ }^{11-16}$ However, only few of the techniques applied to these studies are able to monitor dynamic interactions in situ, within a fluid environment that may be tailored to model likely conditions encountered in vivo. ${ }^{17}$

Surface plasmon resonance (SPR) $)^{14,17-19}$ has been recognized as a powerful and advantageous label-less method for studying the thermodynamics and kinetics of interfacial interactions in situ. ${ }^{17}$ SPR modes are hybrid modes of the free electrons of a metal and the electromagnetic field. These modes are confined at a metal-dielectric interface, propagate along it, and are extremely sensitive to interfacial changes in dielectric properties, such as those that arise from adsorption, binding or cell-adhesion events. Thin metal films are necessary to support surface plasmons in sensing applications and the majority of commercial instruments currently used for 
quantitative analysis and screening rely on this type of sensing elements. More recently, nanoplasmonic sensing (NPS) based on the physical phenomenon of localized surface plasmon resonance (LSPR) has emerged as a valuable alternative. In LSPR modes light interacts with particles much smaller than the incident wavelength leading to a hybrid confined mode with a characteristic resonant frequency that depends on optical properties of metal and dielectric, and on nanoparticle geometry. ${ }^{20-23}$ LSPR can be leveraged for biological and chemical sensing by monitoring the wavelength shifts of the characteristic resonance, which take place in response to changes in the local refractive index. ${ }^{20-21}$ Compared to conventional SPR, NPS offers advantages such as a lower sensitivity to bulk changes, the ability to modulate the optical operating range through careful nanostructure design, and greater hardware flexibility and simplicity. ${ }^{20}$ Technical developments in the large scale fabrication of nanoscale metallic structures have been key to the exploitation of LSPR, resulting in increased interest in LSPR sensing strategies. ${ }^{20,22-23}$

This work describes the application of NPS to in situ studies of the carbon-bio interface. SPR methods have found limited applications so far for the study of interfacial events at carbon surfaces in biological media because of the requirement of metal surfaces for sustaining SPR modes. Lockett et $a .^{24}$ demonstrated however that it is possible to sustain SPR modes at the carbon-liquid interface via deposition of thin carbon coatings of optimized thickness onto Au SPR sensors, a strategy that had previously proved viable for the study of interactions at

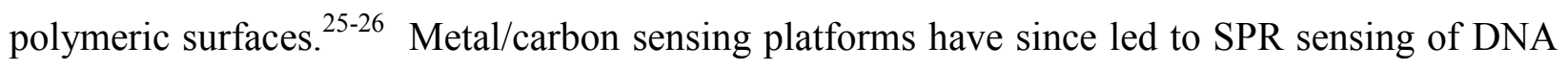
binding, ${ }^{24,27}$ cell binding, ${ }^{28}$ protein adsorption ${ }^{12,29-30}$ and immunosensing ${ }^{31}$ at carbon surfaces whereby the authors demonstrated that SPR is a viable method for monitoring carbonbiomolecule interactions. However, few experimental studies report a comparison of different carbon surfaces under comparable conditions, partly because of the broad variability of the 
optical properties of carbon materials which adds complexity to the analysis of SPR data from metal/carbon/biomolecule multilayers. Notably, Saitoh and co-workers presented a comparative SPR study of albumin adsorption at hydrogenated carbons with different chemical composition, ${ }^{29}$ but pointed out that a quantitative determination of the thickness of adsorbed layers from angle shift data using Fresnel equations is challenging due to strong correlation in the multilayer model between thickness and optical constants.

In this work, NPS was used in combination with ellipsometry and computational methods to estimate the thickness of the protein layer at two carbon substrates with differing optical properties. To our knowledge NPS has not been used for the study of interactions at carbon coatings; herein we apply a recently reported NPS method developed by Kasemo et al. based on Au nanodisk sensing elements. ${ }^{32,33-34}$ Studies of interfacial chemistry on this NPS platform have been typically carried using sensors coated with thin films of dielectrics, such as metal oxides or silica, which ensure a homogeneous surface chemistry and allow flexibility in terms of the chemical reactions under study. ${ }^{35}$ Previous work by Cho and co-workers using biomolecules has reported a detailed study of the effect of dielectric coatings on interfacial chemistry and sensitivity. ${ }^{34}$ In this work, the applicability of these nanostructured sensors to the study of protein adsorption at carbon surfaces in real time is demonstrated. Carbon coatings differ from typical oxide spacer layers, as their optical properties can vary significantly with electronic behavior that spans the semimetallic-semiconductor-insulator range. ${ }^{36}$ Two types of carbon with differing composition were chosen to investigate the effect of carbon chemistry on protein adsorption: a$\mathrm{C}$, a graphitic carbon, and a-C:H, a hydrogenated, polymer-like and $\mathrm{sp}^{3}$-rich, carbon. The two plasma proteins used, albumin and fibrinogen, were chosen because of their importance for understanding the response of biomaterials after implantation. A quantitative modelling of NPS 
results was carried out using the finite difference time domain (FDTD) method for determining protein layer thickness and finally, predictions from FDTD methods were correlated to results from complementary spectroscopic and microscopic methods.

\section{Experimental Methods}

Chemicals and Materials. Ethylene glycol (99.8\%), methanol (semiconductor grade), Bovine serum albumin (BSA, $\geq 96 \%$ ), Fibrinogen from bovine plasma (Fib, 65-85\% protein) and phosphate saline buffer tablets (PBS, 0.01 M, 0.0027 KCl M and $0.137 \mathrm{NaCl} \mathrm{M} \mathrm{pH} \mathrm{7.4)} \mathrm{were}$ purchased from Sigma and used without further purification. B-doped Si wafers were purchased from MicroChemicals (5-10 Ohms) and NPS sensor chips were purchased from Insplorion AB. Millipore water was used for all experiments.

Substrate Preparation. Amorphous carbon films were prepared via DC magnetron sputtering (Torr International, Inc.) at a base pressure $\leq 2 \times 10^{-6} \mathrm{mbar}$ and a deposition pressure of $7 \times 10^{-3}$ mbar, as previously described..$^{37}$ Two distinct films were prepared by varying the $\mathrm{H}_{2} / \mathrm{Ar}$ gas ratio: one type of film was sputtered using $\mathrm{Ar}$ and shall be referred to as a-C from here onwards, the second type of film was sputtered using $10 \% \mathrm{H}_{2}$ in Ar resulting in a hydrogen doped material which is referred to as a-C:H. Silicon wafers were cleaned in piranha solution prior to deposition $\left(\mathrm{H}_{2} \mathrm{SO}_{4}: \mathrm{H}_{2} \mathrm{O}_{2}\right.$ in a 3:1 ratio - WARNING: Piranha solution is a strong oxidant and reacts violently with organic materials and presents an explosion danger; all work should be performed under a fume hood). For spectroscopic ellipsometry (SE) and atomic force microscopy (AFM) characterization, samples were deposited on clean Si wafers. For infrared reflectance absorbance spectroscopy (IRRAS) measurements, Si wafers were first coated with an optically thick $(\sim 450 \mathrm{~nm})$ Ti layer via DC magnetron sputtering, ${ }^{38}$ and subsequently with either a-C or a-C:H films of approximately 70 and $40 \mathrm{~nm}$ thickness, respectively. For NPS 
measurements, sensor chips were cleaned under UV/ozone for $1 \mathrm{~h}$, rinsed with methanol and dried with argon, prior to deposition of a-C and $\mathrm{a}-\mathrm{C}: \mathrm{H}$; the thickness of the layers was determined to be $(10.1 \pm 0.5) \mathrm{nm}$ and $(12.2 \pm 2.1) \mathrm{nm}$ for a-C and a-C:H (95\% C.I.), respectively (see Supporting Information).

Characterization Methods. Spectroscopic Ellipsometry (SE) was carried out using an alphaSETM ellipsometer (J.A. Woolam Co.). Carbon films were deposited on clean Si wafers and measured at $65^{\circ}, 70^{\circ}, 75^{\circ}$ incidence angle over the $370-900 \mathrm{~nm}$ range; SE data was then fitted using the CompleteEASE ${ }^{\circledR}$ software package using a three layer model to account for Si, carbon and air phases (see Supporting Information). ${ }^{38-39}$ IRRAS spectra were collected on a Fourier Transform Infrared (FTIR) spectrometer (Tensor 27, Bruker) equipped with a Mercury Cadmium Telluride (MCT) detector, a specular reflectance accessory (VeeMax II), and a Zinc Selenide polarizer. Spectra were taken at $80^{\circ}$ incidence using p-polarized light; 100 spectra were collected at $4 \mathrm{~cm}^{-1}$ resolution using a bare substrate as background. All spectra reported in this work were baseline corrected using commercial FTIR software (WinFIRST). To account for differences in optical enhancement in IRRAS peaks on a-C and a-C:H, spectral intensities were normalized by the intensity of the $\mathrm{C}=\mathrm{O}$ stretching absorbance of a reference $5.7 \mathrm{~nm}$ poly(methyl methacrylate) (PMMA) layer. UV-Vis transmission measurements of plasmon extinction spectra were obtained in air for bare and carbon coated NPS sensors over the wavelength range 500-800 nm at $1 \mathrm{~nm}$ resolution (Shimadzu UV-2401 PC). Thickness and surface roughness measurements were carried out via AFM (Asylum Research) using Au-coated silicon cantilevers (NT-MDT) in tapping mode (1 Hz and 512 scan lines).

Nanoplasmonic sensing (NPS). Measurements of protein adsorption were conducted using an XNano instrument (Insplorion AB, Gothenburg, Sweden). Ensemble-averaged recordings of the 
resonance peak were collected in optical transmission mode. Glass sensor chips (Insplorion AB) with deposited gold nanodisks (50 nm radius, $20 \mathrm{~nm}$ thickness, $8 \%$ surface coverage) fabricated by hole-mask colloidal lithography, were coated with sputter-deposited a-C or a-C:H layers as described above and mounted in an optical flow cell for in situ measurements. Sample solutions were flowed through the measurement chamber via a peristaltic pump at a continuous rate of 50

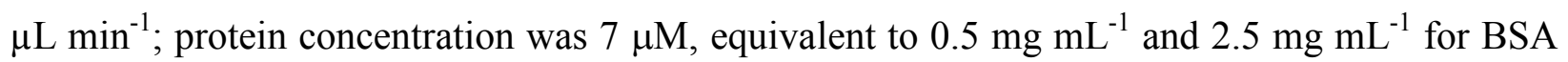
and Fib, respectively. The bulk refractive index of reference ethylene glycol/water solutions used for calibrations was determined using a refractometer (PAL-1, ATAGO Co., Tokyo, Japan).

Computational modelling of sensor response. The Maxwell equations were solved using the finite difference time domain (FDTD) method, as implemented in the commercial package FDTD Solutions from Lumerical. The optical response of the nanostructured sensing elements comprising the coated nanodisk and the substrate were modelled based on their complex refractive index, with values for the specific a-C and a-C:H layers being experimentally determined via spectroscopic ellipsometry.

\section{Results and Discussion}

Carbon films used in our experiments were deposited via magnetron sputtering using Ar and $\mathrm{H}_{2} / \mathrm{Ar}$ as deposition gases; these films had previously been characterized via a combination of spectroscopic methods. ${ }^{37}$ Briefly, a-C and a-C:H films consist of approximately $80 \%$ and $17 \%$ trigonally bonded carbon $\left(\mathrm{sp}^{2}\right.$ centers), respectively, as estimated via X-ray photoelectron spectroscopy (XPS) and Raman spectroscopy. These films also contain oxidized groups resulting in a 7-9\% $\mathrm{O} / \mathrm{C}$ atomic ratio for both $\mathrm{a}-\mathrm{C}$ and $\mathrm{a}-\mathrm{C}: \mathrm{H}$, as determined via XPS. The a-C films are highly graphitic and possess good conductivity, whereas a-C:H films are insulating. ${ }^{37}$ 
Spectroscopic ellipsometry was used to determine optical properties of the films. The optical constants, obtained as discussed in the Supporting Information and previous work, ${ }^{38}$ were consistent with the difference in graphitic content between the two materials. The Tauc gap and absorption coefficients were $\mathrm{E}_{\mathrm{T}}=0.66 \pm 0.01 \mathrm{eV}$ and $\alpha(632 \mathrm{~nm})=(77.2 \pm 0.7) \times 10^{3} \mathrm{~cm}^{-1}$ for a-C $(95 \%$ C.I. $)$ and $\mathrm{E}_{\mathrm{T}}=1.77 \pm 0.01 \mathrm{eV}$ and $\alpha(632 \mathrm{~nm})=(5.08 \pm 0.17) \times 10^{3} \mathrm{~cm}^{-1}$ for a-C:H $(95 \%$ C.I.), thus indicating that a-C films are more metal-like and optically absorbing than a-C:H. ${ }^{36,40}$ The real part of the refractive index was also different for the two materials: $n(632 \mathrm{~nm})=2.117 \pm$ 0.003 for a-C $(95 \%$ C.I.), which is consistent with values obtained for graphitic amorphous carbons, whereas $n(632 \mathrm{~nm})=1.672 \pm 0.003$ for a-C:H $(95 \%$ C.I. $)$, consistent with a low density highly hydrogenated amorphous carbon film. ${ }^{36,41}$

The two types of carbon material were used for in situ studies of protein adsorption using NPS methods. Sensor chips consisting of a glass substrate with nanofabricated gold nanodisks were coated by layers of either a-C or a-C:H, as shown in the schematic in Figure 1; the carbon layers were confirmed to be continuous at the thicknesses of $(10.1 \pm 0.5) \mathrm{nm}$ and $(12.2 \pm 2.1) \mathrm{nm}$ used for NPS experiments (see Supporting Information). The gold nanodisks are randomly distributed on the glass substrate with $8 \%$ surface coverage, ${ }^{33}$ yielding an average disk to disk separation large enough for the discs to be considered independent from each other. The sensors were mounted in a flow cell and the plasmon excitation associated with the gold nanodisks was measured in transmittance mode; the center of mass of the excitation peak was monitored as a function of time during flow experiments. Figure 1 shows typical plasmon resonance peaks obtained in air for a bare $\mathrm{Au}$ sensor, and for $\mathrm{Au} / \mathrm{a}-\mathrm{C}$ and $\mathrm{Au} / \mathrm{a}-\mathrm{C}: \mathrm{H}$ coated sensors. The presence of $\mathrm{a} \sim 10 \mathrm{~nm}$ thick carbon coating does not suppress the plasmon resonance, despite the carbon 


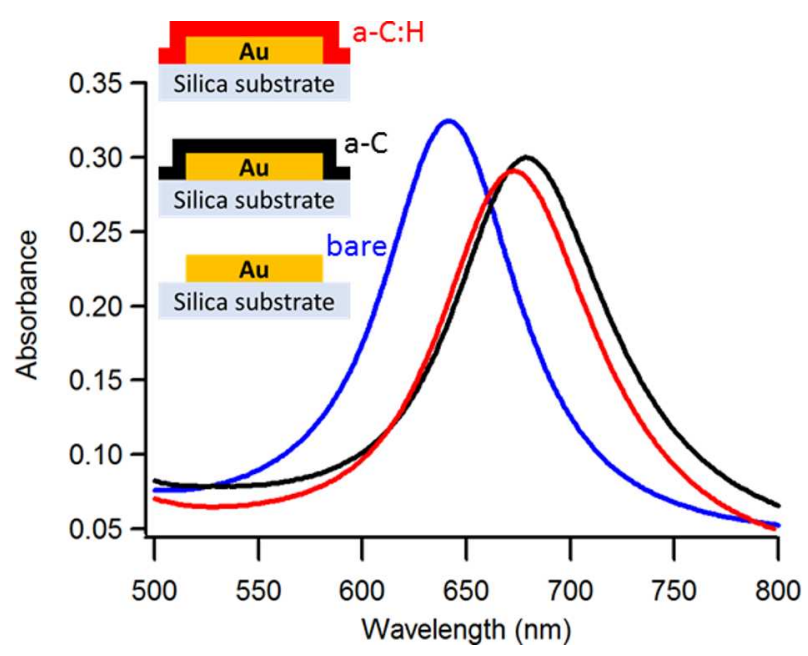

Figure 1. Plasmon absorbance spectrum in air recorded at bare (blue line), a-C (black line) and $\mathrm{a}-\mathrm{C}: \mathrm{H}$ (red line) coated sensors. The inset at the top left of the figure shows schematics of the nanodisk structures that result in the LSPR spectra.

being a continuous layer, however it has an effect on both resonance peak position and fullwidth-at-half-maximum (fwhm), which can in turn affect the sensitivity of the LSPR modes.

The effect of carbon coatings on the sensitivity of LSPR chips was investigated using a combination of computational and experimental methods. The wavelength of maximum extinction, $\lambda_{\max }$, and the fwhm are sensitive to changes in the dielectric properties of the medium at the nanodisk interface. FDTD simulations were used to calculate the plasmon extinction of coated Au nanodisks: the geometry used in the simulations is as shown in Figure 1, with the Au nanodisk possessing $50 \mathrm{~nm}$ radius and $20 \mathrm{~nm}$ thickness. Due to the low surface coverage it is assumed that nanodisks are effectively decoupled, and a single nanodisk element was thus considered in all simulations. This assumption was found to be satisfactory as will be discussed below. The carbon coating was considered as a conformal, uniform layer of $10 \mathrm{~nm}$ and $12 \mathrm{~nm}$ for a-C and a-C:H, respectively, which corresponds to the experimentally determined thickness for 
each layer. The optical constants for Au were obtained from Johnson and Christy, ${ }^{42}$ the refractive index for the glass substrate was real and constant at 1.459 over the wavelength range explored, and those of a-C and a-C:H films were obtained from experimental ellipsometry results (see Supporting Information). Figures $2 \mathrm{a}$ and $2 \mathrm{~b}$ show the logarithm of the absolute value of the total field distribution in the xz-plane, for a single nanodisk coated with a-C and a-C:H, respectively. The exciting electromagnetic field is normally incident on the top of the nanostructure and the excitation wavelengths are chosen to coincide with the maxima of the LSPR extinction in each case. We observe that the field is enhanced by up to two orders of magnitude at the edges of the Au nanodisk. The field around the Au nanodisk extends further beyond the carbon coating for a$\mathrm{C}: \mathrm{H}$, compared to a-C, in agreement with the imaginary part of the refractive index being higher for a-C than for a-C:H. A simulation of the effect of carbon coating thickness on position and shape of the plasmon extinction shows that the presence of both carbon coatings leads to a red shift in the plasmon position and an increase in the fwhm of the peak (see Supporting Information). However, the peak shift and peak broadening effects are significantly more pronounced for a-C than for a-C:H, in agreement with the former being the material with higher optical losses. The field distribution observed in Figures $2 \mathrm{a}$ and $2 \mathrm{~b}$ results from plane-wave excitation of the LSPR dipole mode. The differences in field distributions observed for a-C and a-C:H coatings suggest that the sensitivity of NPS elements to adsorption/binding might be significantly affected, depending on the type of carbon used to coat the sensor. A simulated calibration experiment was thus carried out, in which the resonance maximum position, $\Delta \lambda_{\max }$, was calculated as a function of the refractive index in the medium surrounding the carbon (medium 1). The refractive index range explored was chosen to be identical to one that could be accessed experimentally using water/ethylene glycol solutions. ${ }^{33-34}$ Figure 2c shows the 
a)

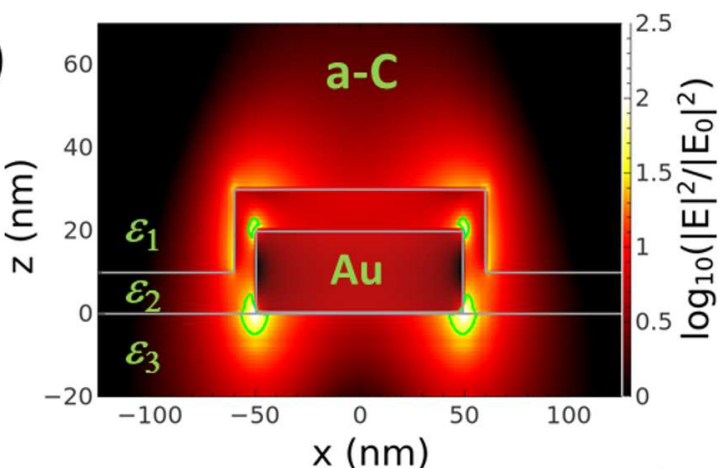

b)

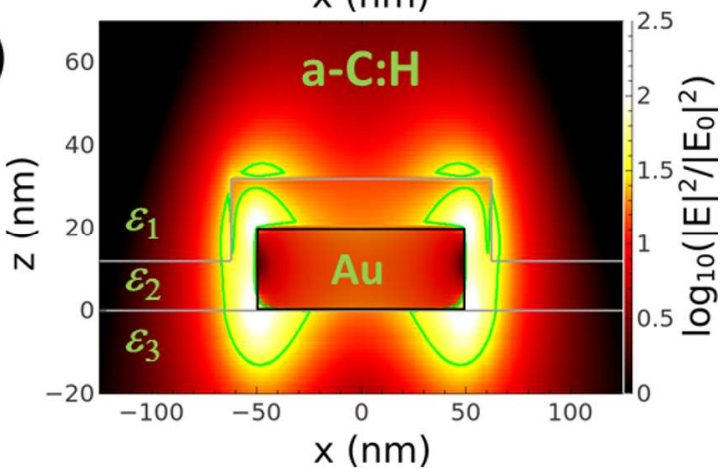

c)

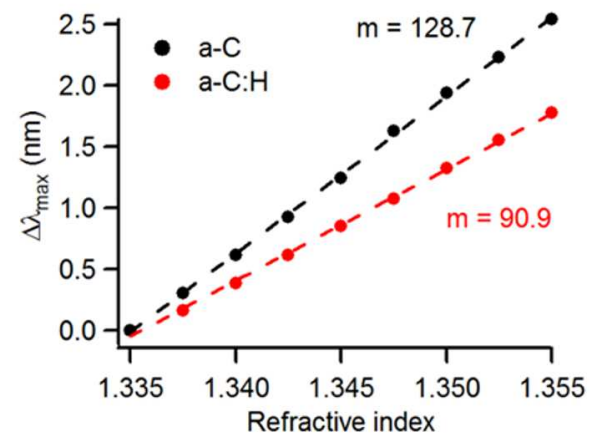

Figure 2. Electric field intensity distribution around isolated nanodisks immersed in PBS obtained via FDTD modelling at the wavelength corresponding to the maximum of the LSPR. The refractive indeces used in the simulation are reported as $\varepsilon_{1}, \varepsilon_{2}$ and $\varepsilon_{3}$ for the aqueous medium $\left(\varepsilon_{1}=1.333\right)$, the carbon coating and the glass substrate, respectively. The green line in the graphs indicates a factor of 30 increase in the electric field intensity. (a) Field distribution around an isolated Au/a-C coated nanodisk at $797 \mathrm{~nm}$; (b) Field distribution around an isolated Au/a-C:H coated nanodisk at $748 \mathrm{~nm}$. (c) Calibration plots obtained via FDTD methods for Au/a-C (black line) and $\mathrm{Au} / \mathrm{a}-\mathrm{C}: \mathrm{H}$ (red line) coated nanodisks.; the slope yielding the analytical sensitivity is reported next to the corresponding curve. 
a)

b)
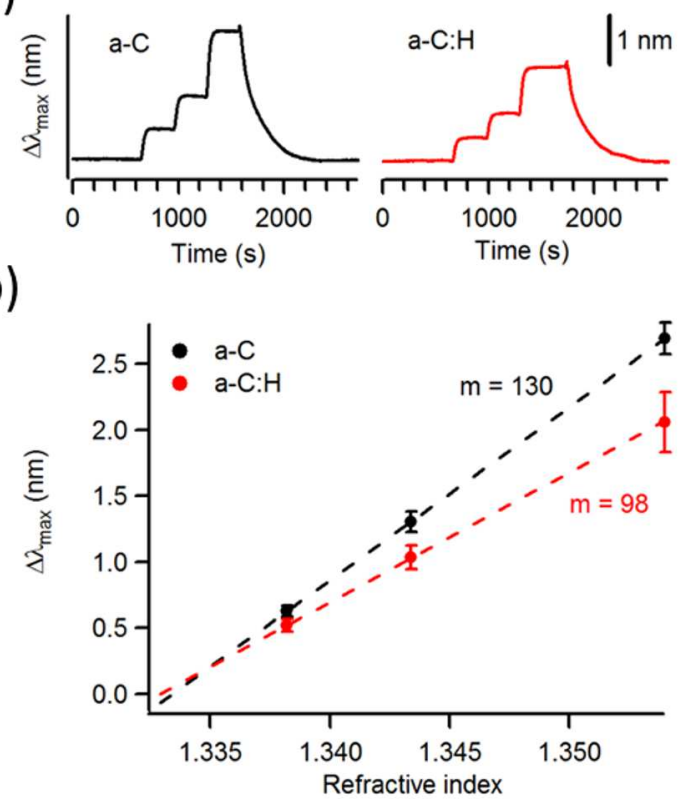

Figure 3. Sensitivity test obtained at a-C (black, left) and a-C:H (red, right) coated sensors. (a) NPS shift $\Delta \lambda_{\max }$ as a function of time measured after water/ethylene glycol solutions of different refractive index are injected into the cell. (b) Calibration plot of measured $\Delta \lambda_{\max } v s$. refractive index of the water/ethylene glycol solution; the slope yielding the analytical sensitivity is reported next to the corresponding curve. Error bars indicate 95\% C.I. calculated from sample size $n=5$ and 3 for a-C and a-C:H, respectively.

calibration plots obtained via FDTD methods for a-C and a-C:H coated sensors. The figure indicates that in both cases the LSPR at the nanodisk is sensitive to changes at the carbon/solution interface. The slopes obtained were $128.7 \mathrm{~nm}$ and $90.9 \mathrm{~nm}$ per unit change in refractive index for a-C and a-C:H, respectively, indicating that the more graphitic film results in higher sensitivity to refractive index changes.

The sensitivity of carbon coated sensors was also investigated experimentally by measuring the shift $\Delta \lambda_{\max } v s$. refractive index of the liquid in the flow cell. Figure 3a shows typical dependence of $\Delta \lambda_{\max }$ as a function of time obtained for a-C and a-C:H coated sensors, as water/ethylene 
glycol solutions of different refractive index are injected into the cell. The staircase response was used to generate a calibration plot as shown in Figure 3b, which shows that the experimental $\Delta \lambda_{\max }$ varies linearly with refractive index. As seen in the calibration plot, changes in the medium refractive index cause a larger peak shift in the optical extinction spectrum of a-C than of a-C:H coated sensors, in agreement with computational predictions. The average experimental slopes were found to be $130 \pm 8 \mathrm{~nm}$ and $98 \pm 12 \mathrm{~nm}$ per unit change in the refractive index, for a-C and $\mathrm{a}-\mathrm{C}: \mathrm{H}$ coated sensors, respectively. The experimentally determined sensitivities therefore compare very well with those obtained via FDTD simulations. This further confirms that the Au nanodisks can be assumed to be decoupled.

In order to evaluate how proteins adsorb at different carbon substrates, both a-C and a-C:H sensors were exposed to buffered protein solutions. All sensors were mounted and calibrated in advance of all measurements using at least three water/ethylene glycol solutions. After calibration, PBS was injected first, followed by the protein solution and a final rinsing step with PBS. The calibration process was repeated at the end of each experiment to exclude any changes to the sensor sensitivity that might arise from adsorbed protein layers. Figure $4 \mathrm{a}$ and $4 \mathrm{~b}$ show plots of $\Delta \lambda_{\max } v s$. time obtained on a-C and a-C:H coated sensors, respectively, after the injection of protein solutions followed by injection of PBS. The full experiment, including the calibration steps, is reported in the Supporting Information. The exposure of carbon coated sensors to protein solutions results in a red shift of the LSPR that stabilizes to a constant value within 15 min. after the injection. Given that the refractive index of the protein solutions was statistically indistinguishable from that of the PBS solution (see Supporting Information), the wavelength shift can be unequivocally attributed to the adsorption of proteins at the carbon surface. The sharper step observed in the case of Fib solutions suggests that adsorption at the carbon surface is 

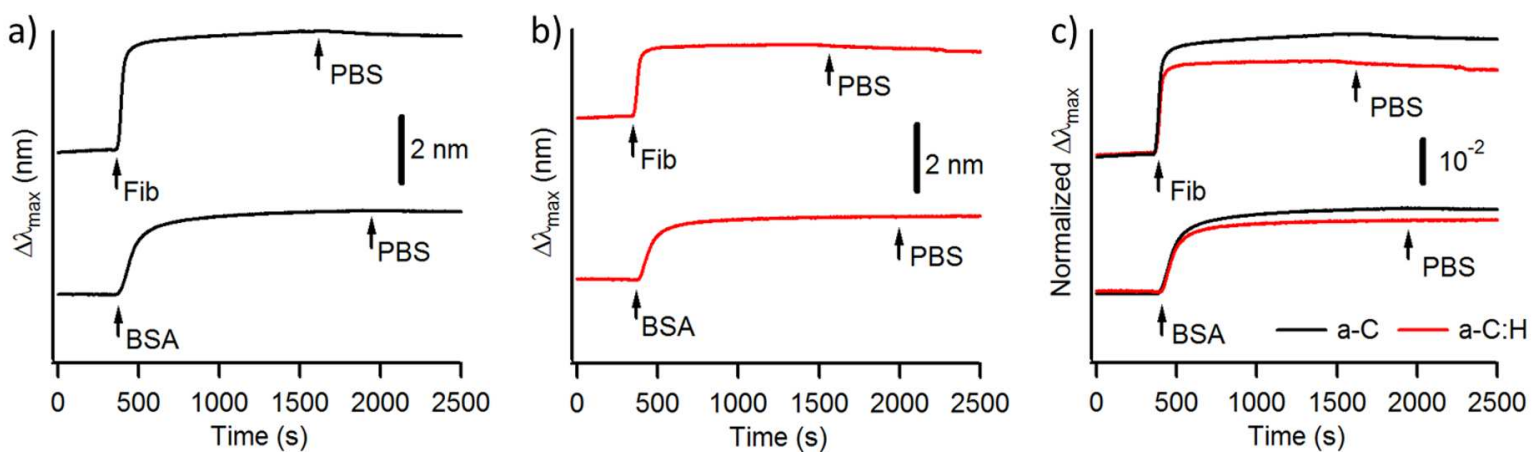

Figure 4. NPS wavelength shift, $\Delta \lambda_{\max }$, as a function of time, measured at (a) a-C and (b) a-C:H coated sensors for in situ protein experiments. (c) Normalized $\Delta \lambda_{\max }$ as a function of time calculated using the initial calibration of the sensor at both a-C (black line) and a-C:H (red line) surfaces. The arrows indicate the time of the injection of BSA, Fib and PBS solutions into the flow cell.

faster for Fib than for BSA; this is also evident from a comparison of the first derivative of the curves (see Supporting Information). Given that the bulk molar concentration is identical, the difference is a result of kinetic control, as a mass transport controlled process should be faster for BSA by a factor of $\sim 1.7$ based on reported diffusion coefficient values of $6 \times 10^{-7} \mathrm{~cm}^{2} \mathrm{~s}^{-1}$ and $2 \times$ $10^{-7} \mathrm{~cm}^{2} \mathrm{~s}^{-1}$ for albumin and fibrinogen, respectively. ${ }^{43}$ Moreover, the absence of any significant change in $\Delta \lambda_{\max }$ after injection of protein-free buffer solution indicates that protein adsorption is irreversible at both a-C and a-C:H surfaces.

The shift of the plasmon resonance is always larger at a-C than at a-C:H coated sensors when comparing adsorption from the same protein solution, however, to compare adsorption at a-C and a-C:H surfaces, the raw signal must be normalized by the experimental sensitivity. The NPS signal of each sensor was thus normalized using the slope of the calibration plot obtained as the first step in each experimental run. This normalization accounts for any differences in alignment across sensors, and for differences in sensitivity to changes in bulk refractive index that result 
from the two types of carbon coating. Figure $4 \mathrm{c}$ shows the normalized $\Delta \lambda_{\max } v s$. time calculated as $\Delta \lambda_{\max } / A$, where $A$ is the slope obtained from the initial calibration of the sensor. A summary of the average normalized $\Delta \lambda_{\max }$ observed for the two carbon surfaces and the two proteins is reported in Table 1. After normalization, results indicate that resonance shifts are slightly greater at a-C than at a-C:H surfaces under the same conditions, thus suggesting that protein adsorption might be more pronounced at a-C than at a-C:H surfaces. For both surfaces the shift obtained for Fib is greater than that observed with BSA, which suggests greater protein adsorption from Fib solutions than from BSA solutions at the same molar concentration, in agreement with previous results obtained using ex situ determinations at a-C surfaces. ${ }^{38}$

FDTD methods were used for the analysis of experimental LSPR shifts to obtain quantitative estimates of the protein layer thickness. The protein layer was simulated as an additional conformal layer on top of the carbon coating, with the same geometry as in Figures $2 \mathrm{a}$ and $2 \mathrm{~b}$. The layer was modelled as a dielectric with a constant real refractive index of $1.465{ }^{44}$ The LSPR wavelength shift, $\Delta \lambda_{\max }$, was simulated for both a-C and a-C:H coated sensors, at various thicknesses of the protein layer, and the normalized $\Delta \lambda_{\max }$ was then calculated using the slopes of the computed calibration plots (Figure 2c). Figure 5 shows the normalized $\Delta \lambda_{\max }$ calculated at different thicknesses of the protein layer. The plot shows that $\Delta \lambda_{\max }$ levels off at large layer thickness. This is in agreement with expectations as the resonance shift should tend to a limit, corresponding to the value obtained for a semi-infinite medium with a refractive index equivalent to that of the protein layer. The experimental data obtained from the NPS measurements was used to estimate the thickness of the protein layer at the sensor surface via interpolation of the curves in Figure 5. The thickness estimates thus obtained are reported in Table 1. The protein film thicknesses obtained from in situ NPS experiments using FDTD- 
Table 1. Summary of results from NPS and AFM measurements.

\begin{tabular}{|c|c|c|c|c|c|}
\hline Surface & Protein & $\begin{array}{l}\text { Normalized } \\
\Delta \lambda_{\max }\left(\times 10^{-2}\right)\end{array}$ & $\begin{array}{l}\text { Modelled } \\
\text { thickness (nm) }\end{array}$ & $\begin{array}{l}\mathrm{RMS}^{a} \\
\text { roughness (nm) }\end{array}$ & $\begin{array}{l}\operatorname{AFM}^{b} \\
\text { thickness (nm) }\end{array}$ \\
\hline \multirow{2}{*}{$\mathrm{a}-\mathrm{C}$} & $\overline{B S A}$ & $2 \pm 0.5$ & 2.3 & 1.05 & $1.0 \pm 0.1$ \\
\hline & Fib & $2.8 \pm 1.1$ & 3.2 & 1.91 & $1.4 \pm 0.1$ \\
\hline \multirow{2}{*}{$\mathrm{a}-\mathrm{C}: \mathrm{H}$} & BSA & $1.4 \pm 0.6$ & 1.8 & 1.22 & $1.3 \pm 0.1$ \\
\hline & Fib & $2.3 \pm 0.5$ & 3.3 & 2.04 & $1.8 \pm 0.1$ \\
\hline
\end{tabular}

Figure 5. Simulated normalized $\Delta \lambda_{\max }$ for a-C (black) and a-C:H (red) coated sensors calculated for various thicknesses of the protein layer using the FDTD method. $\Delta \mathrm{z}$ step measured after a contact mode experiment.

generated calibration plots are in the range $1.8-3.3 \mathrm{~nm}$; in the case of BSA the estimated adsorbed layer is thinner for a-C:H than for a-C, however, in the case of Fib, the adsorbed layer thickness is similar for both types of carbon surfaces. Given that the same refractive index was assumed for BSA and Fib layers, the thickness ratio provides a measure of relative mass density for the two proteins. ${ }^{45}$ Using a ratio of molar mass to molar refractivity of 4.14 typical of 
proteins and Cuypers one-component model, ${ }^{46}$ the estimated mass density for Fib is approximately $3.8 \mathrm{mg} \mathrm{m}^{-2}$ on both surfaces, whereas that of BSA is 2.1 and $2.6 \mathrm{mg} \mathrm{m}^{-2}$ on a-C:H and a-C, respectively. In the case of both carbon materials the mass density of Fib was therefore found to be higher than that of the BSA layer.

In situ experiments show that in the case of BSA, a globular protein, the adsorption is slightly higher on a-C vs. a-C:H surfaces. a-C:H displays lower hydrophilicity compared to a-C based on water contact angle measurements; ${ }^{47}$ multisolvent contact angle determinations (see Supporting Information) shows that the surface free energy of a-C:H is $58.4 \mathrm{~mJ} \mathrm{~m}^{-2}$, lower than that of a-C. Estimated BSA layer thicknesses in Table 2 are therefore consistent with both wetting and surface free energy comparisons, as it has been empirically observed that in the range 20-65 mJ $\mathrm{m}^{-2}$, lower surface free energy translates into reduced protein adsorption. ${ }^{48}$ It is likely however that this is not the only mechanism at the origin of the observed differences, as the adsorption of proteins at surfaces is a complex process involving long-range interactions, multiple adsorbate conformations and conformational changes at the surface over multiple timescales. ${ }^{43}$, $49-50$ Recently, Urbassek and co-workers ${ }^{51}$ carried out molecular dynamic simulations of insulin, a small globular protein, adsorbed at graphite surfaces, and examined the effect of immobilized ethane, a hydrocarbon, on the adsorption process. The presence of a hydrocarbon was found to significantly reduce protein-surface interaction energy values and, consequently, protein denaturation at hydrocarbon-covered surfaces. The two surfaces used in our experiments range from a graphite-like surface $(\mathrm{a}-\mathrm{C})$ to a hydrocarbon-like surface, rich in $\mathrm{C}-\mathrm{H}$ bonds $(\mathrm{a}-\mathrm{C}: \mathrm{H}){ }^{37}$ Based on Urbassek's results it is therefore reasonable to expect reduced protein adsorption at a$\mathrm{C}: \mathrm{H}$ compared to a-C. 
In situ experiments also reveal higher protein surface coverages when using Fib compared to BSA independently of the surface examined. Fib is known to form irreversibly adsorbed layers with a wide range of surface density values, but its mechanism of adsorption is still highly debated. ${ }^{52}$ Fib has a higher molecular weight than BSA, it possesses multiple domains and a hinged rod-like shape. Its anisotropy opens the possibility of both side-on and end-on surface approaches, ${ }^{49,53}$ and previous experiments of Fib on $\mathrm{Au},{ }^{53}$ silica, ${ }^{54}$ polymers and mica ${ }^{55}$ have proposed formation of mixed side-on/end-on layers. The estimated mass density for Fib of 3.8 $\mathrm{mg} \mathrm{m}^{-2}$ found in our experiments is in excellent agreement with limiting coverages observed by other groups under similar conditions. ${ }^{52,54}$ However, it is approximately double what is expected for closed packed side-on adsorbates (1.4-2.1 $\left.\mathrm{mg} \mathrm{m}^{-2}\right) .{ }^{54,56}$ This suggests that on a-C and a-C:H Fib might form mixed side-on/end-on layer as proposed for other surfaces. Minton has previously demonstrated via simulations that faster adsorption rates can be expected from end-on vs. side-on adsorbates, which are consistent with faster adsorption observed for Fib in our experiments. Relative to side-on adsorbates, end-on conformation results in weaker proteinsurface interactions, which might also partially contribute to the insensitivity of the Fib thickness to the type of carbon surface chemistry. Based on the current experiments alone it is not possible to distinguish formation of mixed side-on/end-on layers from side-on multilayer formation, however experiments at lower protein concentrations, and/or using complementary techniques to probe adsorbate packing might distinguish between these two possible modes of adsorption.

The adsorbed protein layers were also characterized via ex situ experiments; Figure 6 shows IRRAS spectra in the region $1900-1300 \mathrm{~cm}^{-1}$ of a-C and a-C:H surfaces after incubation in BSA and Fib solutions followed by rinsing. Spectra exhibit the characteristic bands of amide groups in polypeptides at $\sim 1675 \mathrm{~cm}^{-1}$ and $\sim 1540 \mathrm{~cm}^{-1}$, assigned to the amide I and II modes, 


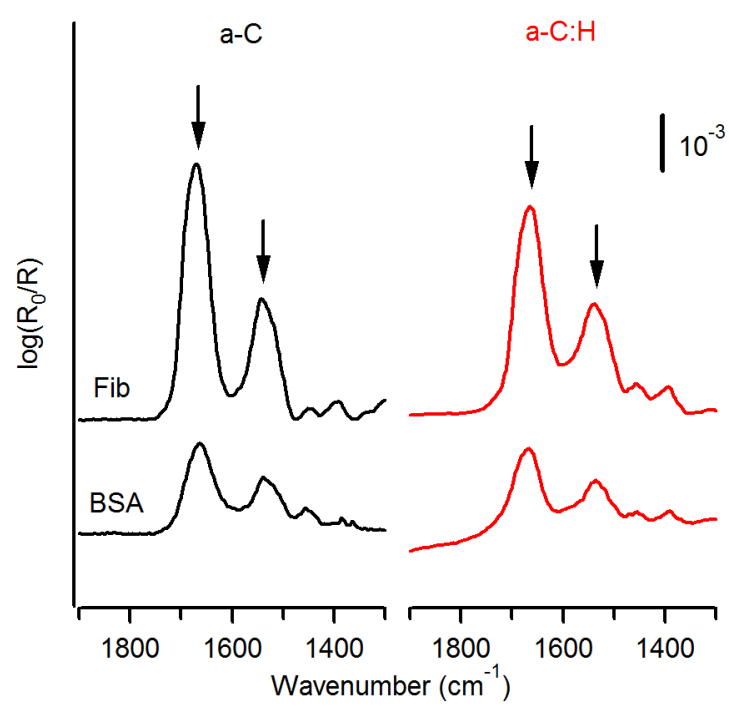

Figure 6. IRRAS spectra of a-C (black, left) and a-C:H (red, right) substrates after $1 \mathrm{~h}$ incubation with BSA (top) and Fib (bottom) solutions. The arrows indicate the peak positions of the amide I and amide II bands. Spectra were baseline corrected and a-C:H peaks were corrected for optical enhancement, as indicated in the experimental section, to facilitate comparison of peak intensities.

respectively. ${ }^{57}$ The two strong broad peaks indicate the presence of the protein layer irreversibly adsorbed at both carbon surfaces, as previously reported by our group in the case of a-C. ${ }^{38}$ The higher intensity obtained for amide peaks in the case of Fib is consistent with NPS results which indicate that under these conditions Fib yields thicker adsorbed layers than BSA. AFM characterization of the films, using previously reported methods. ${ }^{38,58-59}$ was used to compare the morphology of protein layers obtained at the carbon surfaces and to understand whether ex situ and in situ determinations of protein layer thickness resulted in comparable results. Carbon surfaces exposed to protein solutions were first imaged in tapping mode; subsequently, a section of the film was removed by scratching the sample with the AFM tip in contact mode; finally, the step created in the organic film was imaged to determine the layer thickness through cross- 

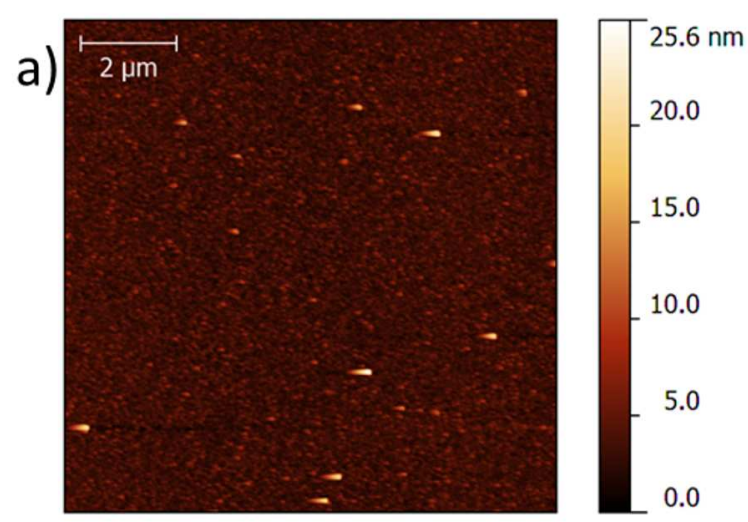

b)

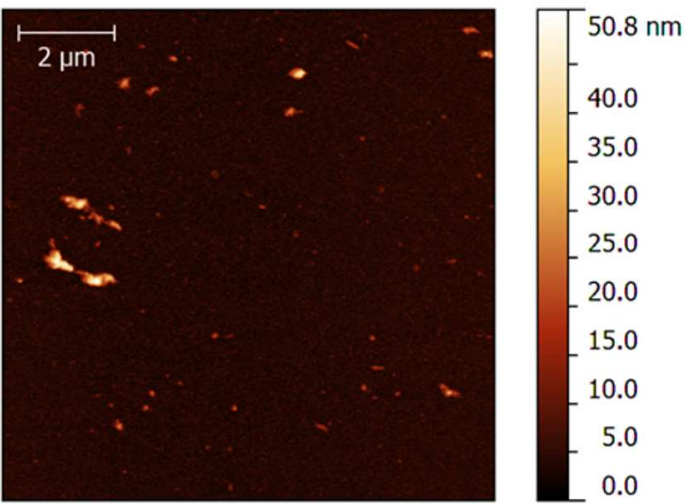

c)

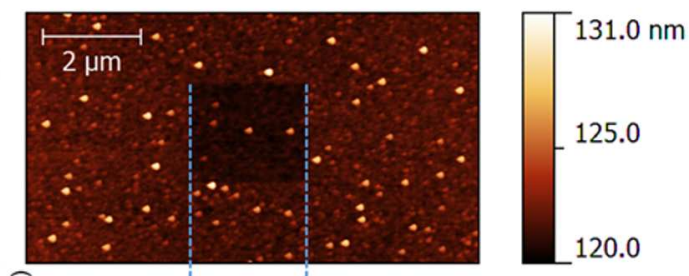

Figure 7. AFM topographic images of a-C:H surfaces after incubation with (a) BSA and (b) Fib solutions; thickness of (c) BSA layer adsorbed at an a-C surface.

section analysis. AFM images reveal that BSA tends to adsorb at both carbon substrates forming smooth layers, whereas Fib tends to form 10-30 nm thick agglomerates (Figure 7a and 7b). Roughness measurements in fact yield higher root mean square (rms) values in the case of Fib at both a-C and a-C:H surfaces, as reported in Table 1. Figure 7c shows an example of a-C after 
incubation in BSA solution, imaged after the scratching process; the height profile across the step is shown in the plot underneath the image. The average height difference of protein layers was found to be in the range 1.0-1.8 $\mathrm{nm}$ in the case of BSA and Fib, respectively, for both a-C and a-C:H substrates (Table 1). These thicknesses are lower than those obtained from in situ NPS measurements, but are consistent with the protein layer undergoing dehydration and compaction after sample drying prior to AFM determinations. When comparing results obtained for the two proteins, AFM measurements also show that Fib yields thicker layers than BSA ( $40 \%$ thicker) at both a-C and a-C:H surfaces, in good agreement with NPS experiments.

\section{Conclusions}

We have studied in situ adsorption of two plasma proteins at different types of carbon surfaces using a NPS method. FDTD simulations that modelled the sensor response based on the nanodisk geometry and carbon optical constants were predictive of the analytical sensitivity. The computation model was, therefore, used to analyses protein adsorption data to determine estimated thicknesses, which were found to be consistent with results obtained via ex situ spectroscopy and microscopy. Mass density estimates calculated from thickness values are in good agreement with limiting protein coverage values previously observed with other techniques. These results suggest that NPS in combination with FDTD analysis are well suited to investigating and comparing protein adsorption at carbons, even in the case of carbon materials with highly dissimilar dielectric properties. We expect the results to be important as a platform for new methodologies for the investigation of the carbon-bio interface. 


\begin{abstract}
ASSOCIATED CONTENT
Spectroscopic ellipsometry analysis; AFM determinations of coating thickness; XPS characterization of coated sensors; extended plots of NPS data in real time; refractive index of solutions used in experiments; additional comparison of experimental and simulated analytical sensitivity of carbon coated sensors; raw data of IRRAS measurements. This material is available free of charge via the Internet at http://pubs.acs.org.
\end{abstract}

\title{
AUTHOR INFORMATION
}

\section{Corresponding Author}

*colavitp@tcd.ie.

\section{Author Contributions}

The manuscript was written mainly by FZ and PEC with contributions from all authors; FZ and JA designed and carried out NPS experimental work; JAB contributed with ellipsometric determinations; GC contributed with XPS data collection; FZ carried out all ex situ characterization; ALB and VK contributed the FDTD simulations. All authors have given approval to the final version of the manuscript.

\section{Funding Sources}

This publication has emanated from research conducted with the financial support of Science Foundation Ireland (SFI) grant No. 12/IP/1273. JAB acknowledges support from the Irish Research Council through Grant No. GOIPG/2014/399; GC acknowledges support from SFI grant No. 13/CDA/2213. ALB and VDK acknowledge support from Science Foundation Ireland (SFI) under grant number 10/IN.1/12975. 


\section{ACKNOWLEDGEMENT}

The authors are grateful to S. Mills and Prof. J.Boland for access to AFM instrumentation. The authors also thank Olof Andersson and Patrik Bjöörn for their generous support through access to NPS instrumentation.

\section{REFERENCES}

1. Roy, R. K.; Lee, K. R., Biomedical applications of diamond-like carbon coatings: A review. J. Biomed. Mater. Res. Part B 2007, 83B (1), 72-84.

2. $\quad$ Stueber, M.; Niederberger, L.; Danneil, F.; Leiste, H.; Ulrich, S.; Welle, A.; Marin, M.; Fischer, H., Surface topography, surface energy and wettability of magnetron-sputtered amorphous carbon (a-c) films and their relevance for platelet adhesion. Adv. Eng. Mater. 2007, 9 (12), 1114-1122.

3. Sydow-Plum, G.; Tabrizian, M., Review of stent coating strategies: clinical insights. Mater. Sci. Technol. 2008, 24 (9), 1127-1143.

4. Anne Thomson, L.; Law, F. C.; Rushton, N.; Franks, J., Biocompatibility of diamond-like carbon coating. Biomaterials 1991, 12 (1), 37-40.

5. Hauert, R., A review of modified DLC coatings for biological applications. Diamond Relat. Mater. 2003, 12 (3-7), 583-589.

6. Ratner, B. D.; Hoffman, A. S.; Schoen, F. J.; Lemons, J. E., Biomaterials Science. 2nd ed.; Elsevier Academic Press: London, 2004.

7. Kasemo, B., Biological surface science. Surf. Sci. 2002, 500 (1-3), 656-677.

8. Kasemo, B.; Lausmaa, J., Surface properties and processes of the biomaterial-tissue interface. Mater. Sci. Eng., C 1994, 1 (3), 115-119.

9. Schwartz, Z.; Boyan, B. D., Underlying mechanisms at the bone-biomaterial interface. $J$. Cell. Biochem. 1994, 56 (3), 340-347.

10. Thevenot, P.; Hu, W.; Tang, L., Surface Chemistry Influences Implant Biocompatibility. Curr. Top. Med. Chem. 2008, 8 (4), 270-280.

11. Yadav, P. K.; McKavanagh, F.; Maguire, P. D.; Lemoine, P., Adsorption of bovine serum albumin on amorphous carbon surfaces studied with dip pen nanolithography. Appl. Surf. Sci. 2011, 258 (1), 361-369. 
12. Takeda, A.; Akasaka, H.; Ohshio, S.; Toda, I.; Nakano, M.; Saitoh, H., Adsorption ability comparison of plasma proteins on amorphous carbon surface. J. Phys. Chem. Solids 2012, 73 (11), 1331-1334.

13. Berlind, T.; Tengvall, P.; Hultman, L.; Arwin, H., Protein adsorption on thin films of carbon and carbon nitride monitored with in situ ellipsometry. Acta Biomater. 2011, 7 (3), 13691378.

14. Lousinian, S.; Logothetidis, S., Optical properties of proteins and protein adsorption study. Microelectron. Eng. 2007, 84 (3), 479-485.

15. Jones, M. I.; McColl, I. R.; Grant, D. M.; Parker, K. G.; Parker, T. L., Protein adsorption and platelet attachment and activation, on TiN, TiC, and DLC coatings on titanium for cardiovascular applications. J. Biomed. Mater. Res. 2000, 52 (2), 413-421.

16. Feng, L.; Andrade, J. D., Protein adsorption on low temperature isotropic carbon. 1. Protein conformational change probed by differential scanning calorimetry. J. Biomed. Mater. Res. 1994, 28 (6), 735-743.

17. Green, R. J.; Frazier, R. A.; Shakesheff, K. M.; Davies, M. C.; Roberts, C. J.; Tendler, S. J. B., Surface plasmon resonance analysis of dynamic biological interactions with biomaterials. Biomaterials 2000, 21 (18), 1823-1835.

18. Kargl, R.; Kahn, M.; Köstler, S.; Reischl, M.; Doliška, A.; Stana-Kleinschek, K.; Waldhauser, W.; Ribitsch, V., Deposition of silicon doped and pure hydrogenated amorphous carbon coatings on quartz crystal microbalance sensors for protein adsorption studies. Thin Solid Films 2011, 520 (1), 83-89.

19. Lousinian, S.; Kalfagiannis, N.; Logothetidis, S., Albumin and fibrinogen adsorption on boron nitride and carbon-based thin films. Mater. Sci. Eng., B 2008, 152 (1-3), 12-15.

20. Willets, K. A.; Duyne, R. P. V., Localized Surface Plasmon Resonance Spectroscopy and Sensing. Annu. Rev. Phys. Chem. 2007, 58 (1), 267-297.

21. Yonzon, C. R.; Jeoung, E.; Zou, S.; Schatz, G. C.; Mrksich, M.; Van Duyne, R. P., A Comparative Analysis of Localized and Propagating Surface Plasmon Resonance Sensors: The Binding of Concanavalin A to a Monosaccharide Functionalized Self-Assembled Monolayer. $J$. Am. Chem. Soc. 2004, 126 (39), 12669-12676.

22. Hutter, E.; Fendler, J. H., Exploitation of Localized Surface Plasmon Resonance. Adv. Mater. (Weinheim, Ger.) 2004, 16 (19), 1685-1706.

23. Anker, J. N.; Hall, W. P.; Lyandres, O.; Shah, N. C.; Zhao, J.; Van Duyne, R. P., Biosensing with plasmonic nanosensors. Nat. Mater. 2008, 7 (6), 442-453.

24. Lockett, M. R.; Weibel, S. C.; Phillips, M. F.; Shortreed, M. R.; Sun, B.; Corn, R. M.; Hamers, R. J.; Cerrina, F.; Smith, L., Carbon-on-Metal Films for Surface Plasmon Resonance Detection of DNA Arrays. J. Am. Chem. Soc. 2008, 130 (27), 8611-8613. 
25. Green, R. J.; Davies, J.; Davies, M. C.; Roberts, C. J.; Tendler, S. J. B., Surface plasmon resonance for real time in situ analysis of protein adsorption to polymer surfaces. Biomaterials 1997, $18(5), 405-413$.

26. Green, R. J.; Davies, M. C.; Roberts, C. J.; Tendler, S. J. B., Competitive protein adsorption as observed by surface plasmon resonance. Biomaterials 1999, 20 (4), 385-391.

27. Zagorodko, O.; Spadavecchia, J.; Serrano, A. Y.; Larroulet, I.; Pesquera, A.; Zurutuza, A.; Boukherroub, R.; Szunerits, S., Highly Sensitive Detection of DNA Hybridization on Commercialized Graphene-Coated Surface Plasmon Resonance Interfaces. Anal. Chem. 2014, 86 (22), 11211-11216.

28. Subramanian, P.; Barka-Bouaifel, F.; Bouckaert, J.; Yamakawa, N.; Boukherroub, R.; Szunerits, S., Graphene-Coated Surface Plasmon Resonance Interfaces for Studying the Interactions between Bacteria and Surfaces. ACS Appl. Mater. Interfaces 2014, 6 (8), 5422-5431.

29. Akasaka, H.; Gawazawa, N.; Suzuki, T.; Nakano, M.; Ohshio, S.; Saitoh, H., Evaluation of protein adsorption on hydrogenated amorphous carbon films by surface plasmon resonance phenomenon. Diamond Relat. Mater. 2010, 19 (10), 1235-1239.

30. Akasaka, H.; Takeda, A.; Suzuki, T.; Nakano, M.; Ohshio, S.; Saitoh, H., Fibrinogen and lysozyme adsorption on amorphous carbon film surface detected by multilayer device from the back side of the film. Diamond Relat. Mater. 2011, 20 (2), 213-216.

31. Singh, M.; Holzinger, M.; Tabrizian, M.; Winters, S.; Berner, N. C.; Cosnier, S.; Duesberg, G. S., Noncovalently Functionalized Monolayer Graphene for Sensitivity Enhancement of Surface Plasmon Resonance Immunosensors. J. Am. Chem. Soc. 2015, 137 (8), 2800-2803.

32. Larsson, E. M.; Langhammer, C.; Zorić, I.; Kasemo, B., Nanoplasmonic Probes of Catalytic Reactions. Science 2009, 326 (5956), 1091-1094.

33. Jackman, J. A.; Spackova, B.; Linardy, E.; Kim, M. C.; Yoon, B. K.; Homola, J.; Cho, N.-J., Nanoplasmonic ruler to measure lipid vesicle deformation. Chem. Commun. 2016, 52 (1), 76-79.

34. Zan, G. H.; Jackman, J. A.; Kim, S.-O.; Cho, N.-J., Biosensors: Controlling Lipid Membrane Architecture for Tunable Nanoplasmonic Biosensing (Small 23/2014). Small 2014, $10(23), 4827-4827$.

35. Langhammer, C.; Larsson, E. M.; Kasemo, B.; Zorić, I., Indirect Nanoplasmonic Sensing: Ultrasensitive Experimental Platform for Nanomaterials Science and Optical Nanocalorimetry. Nano Lett. 2010, 10 (9), 3529-3538.

36. Robertson, J., Diamond-like amorphous carbon. Mater, Sci. Eng. R 2002, 37 (4-6), 129281. 
37. Cullen, R. J.; Jayasundara, D.; Soldi, L.; Cheng, J.; Dufaure, G.; Colavita, P. E., Spontaneous grafting of nitrophenyl groups on amorphous carbon thin films: A structurereactivity investigation. Chem. Mater. 2012, 24 (6), 1031-1040.

38. Zen, F.; Angione, M. D.; Behan, J. A.; Cullen, R. J.; Duff, T.; Vasconcelos, J. M.; Scanlan, E. M.; Colavita, P. E., Modulation of Protein Fouling and Interfacial Properties at Carbon Surfaces via Immobilization of Glycans Using Aryldiazonium Chemistry. Sci. Rep. 2016, $6,24840$.

39. Behan, J. A.; Stamatin, S. N.; Hoque, M. K.; Ciapetti, G.; Zen, F.; Esteban-Tejeda, L.; Colavita, P. E., A Combined Optoelectronic and Electrochemical Study of Nitrogenated Carbon Electrodes. J. Phys. Chem. C 2017, 121 (12), 6596-6604.

40. Silva, S. R. P., Properties of amorphous carbon. 1st ed.; INSPEC, Inc. The Institution of Electrical Engineers: London, 2003.

41. Schwarz-Selinger, T.; von Keudell, A.; Jacob, W., Plasma chemical vapor deposition of hydrocarbon films: The influence of hydrocarbon source gas on the film properties. J. Appl. Phys. 1999, 86 (7), 3988-3996.

42. Johnson, P. B.; Christy, R. W., Optical Constants of the Noble Metals. Phys. Rev. B 1972, $6(12), 4370-4379$.

43. Andrade, J. D.; Hlady, V., Plasma Protein Adsorption: The Big Twelvea. Ann. N. Y. Acad. Sci. 1987, 516 (1), 158-172.

44. Goyal, D. K.; Subramanian, A., In-situ protein adsorption study on biofunctionalized surfaces using spectroscopic ellipsometry. Thin Solid Films 2010, 518 (8), 2186-2193.

45. Vörös, J., The Density and Refractive Index of Adsorbing Protein Layers. Biophys. J. 2004, 87 (1), 553-561.

46. Cuypers, P. A.; Corsel, J. W.; Janssen, M. P.; Kop, J. M.; Hermens, W. T.; Hemker, H. C., The adsorption of prothrombin to phosphatidylserine multilayers quantitated by ellipsometry. J. Biol. Chem. 1983, 258 (4), 2426-31.

47. Vasconcelos, J. M.; Zen, F.; Stamatin, S. N.; Behan, J. A.; Colavita, P. E., Determination of surface $\zeta$-potential and isoelectric point of carbon surfaces using tracer particle suspensions. Surf. Interface Anal. 2017, DOI: 10.1002/sia.6223.

48. Baier, R., Surface behaviour of biomaterials: The theta surface for biocompatibility. $J$. Mater. Sci.: Mater. Med. 2006, 17 (11), 1057-1062.

49. Minton, A. P., Adsorption of Globular Proteins on Locally Planar Surfaces. II. Models for the Effect of Multiple Adsorbate Conformations on Adsorption Equilibria and Kinetics. Biophys. J. 1999, 76 (1), 176-187. 
50. Fang, F.; Szleifer, I., Kinetics and Thermodynamics of Protein Adsorption: A Generalized Molecular Theoretical Approach. Biophys. J. 2001, 80 (6), 2568-2589.

51. Mücksch, C.; Rösch, C.; Müller-Renno, C.; Ziegler, C.; Urbassek, H. M., Consequences of Hydrocarbon Contamination for Wettability and Protein Adsorption on Graphite Surfaces. $J$. Phys. Chem. C 2015, 119 (22), 12496-12501.

52. Adamczyk, Z.; Barbasz, J.; Cieśla, M., Mechanisms of Fibrinogen Adsorption at Solid Substrates. Langmuir 2011, 27 (11), 6868-6878.

53. Dyr, J. E.; Tichý, I.; Jiroušková, M.; Tobiška, P.; Slavík, R.; Homola, J.; Brynda, E.; Houska, M.; Suttnar, J., Molecular arrangement of adsorbed fibrinogen molecules characterized by specific monoclonal antibodies and a surface plasmon resonance sensor. Sens. Actuators $B$ Chem. 1998, 51 (1-3), 268-272.

54. Malmsten, M., Ellipsometry Studies of Protein Layers Adsorbed at Hydrophobic Surfaces. J. Colloid Interface Sci. 1994, 166 (2), 333-342.

55. Cieśla, M.; Adamczyk, Z.; Barbasz, J.; Wasilewska, M., Mechanisms of Fibrinogen Adsorption at Solid Substrates at Lower pH. Langmuir 2013, 29 (23), 7005-7016.

56. Adamczyk, Z.; Barbasz, J.; Cieśla, M., Kinetics of Fibrinogen Adsorption on Hydrophilic Substrates. Langmuir 2010, 26 (14), 11934-11945.

57. Socrates, G., Infrared and Raman Characteristic Group Frequencies: Tables and Charts. John Wiley \& Sons: 2001.

58. Brooksby, P. A.; Downard, A. J., Electrochemical and Atomic Force Microscopy Study of Carbon Surface Modification via Diazonium Reduction in Aqueous and Acetonitrile Solutions. Langmuir 2004, 20 (12), 5038-5045.

59. Anariba, F.; DuVall, S. H.; McCreery, R. L., Mono- and Multilayer Formation by Diazonium Reduction on Carbon Surfaces Monitored with Atomic Force Microscopy "Scratching". Anal. Chem. 2003, 75 (15), 3837-3844. 

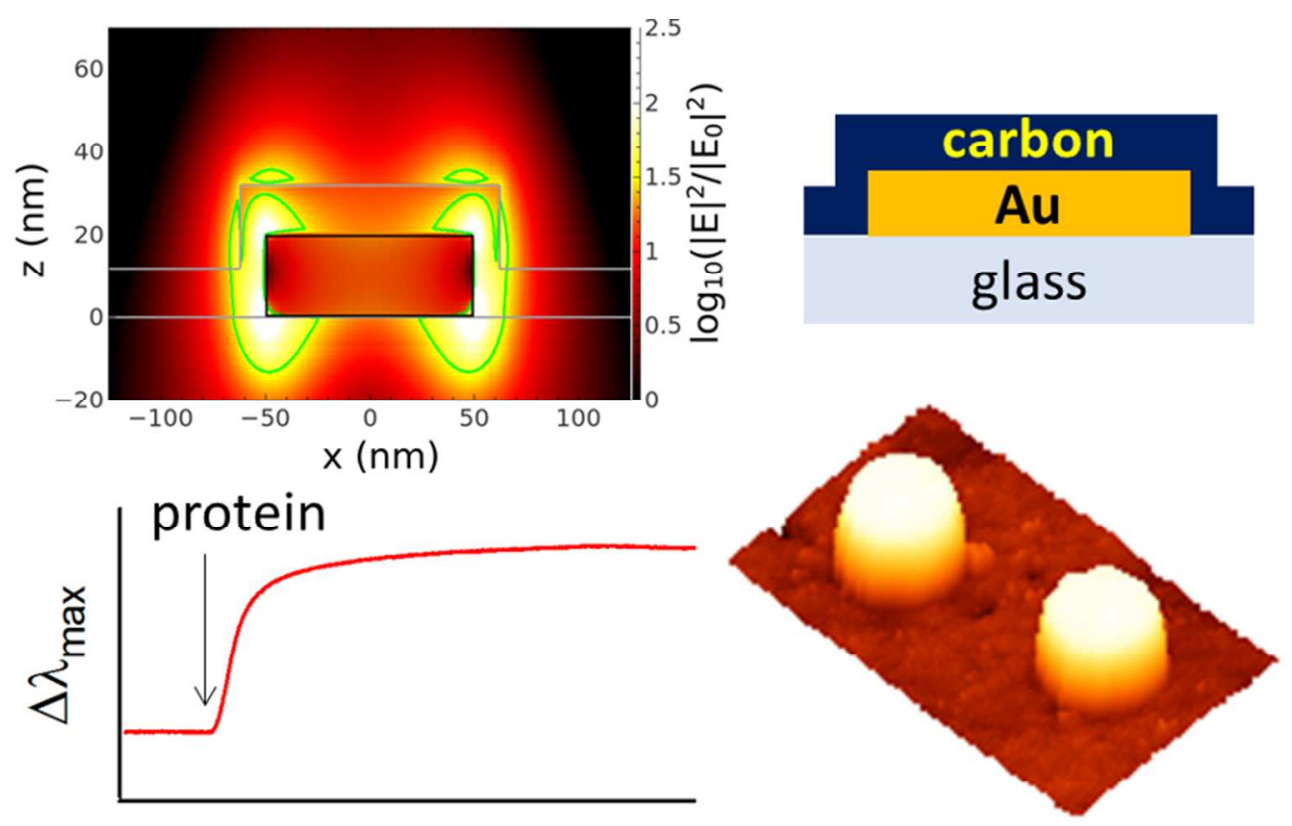

Time (s)

Table of Contents Graphic

$73 \times 50 \mathrm{~mm}(300 \times 300$ DPI $)$ 


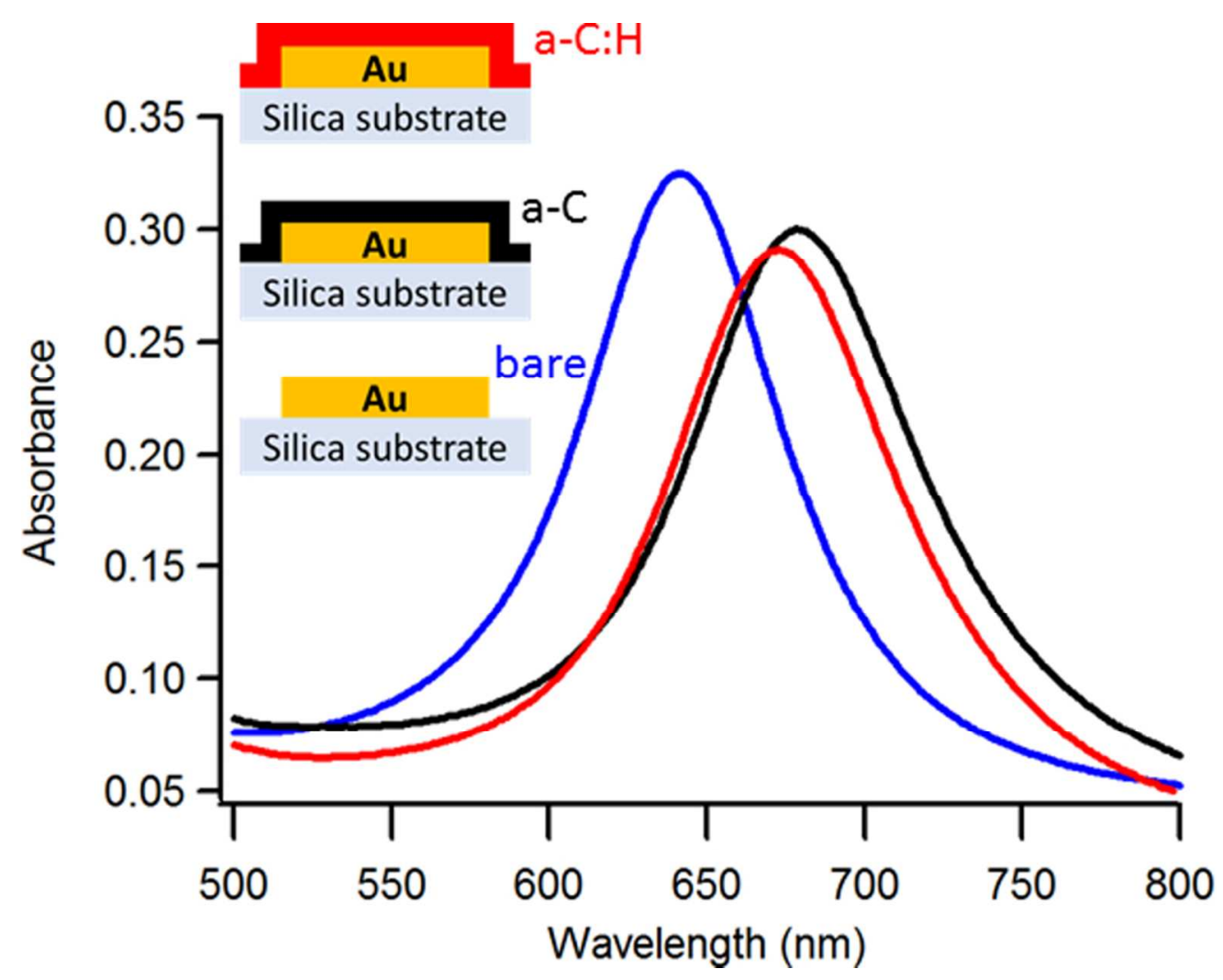

Figure 1. Plasmon absorbance spectrum in air recorded at bare (blue line), a-C (black line) and a-C:H (red line) coated sensors. The inset at the top left of the figure shows schematics of the nanodisk structures that result in the LSPR spectra.

$80 \times 63 \mathrm{~mm}(300 \times 300$ DPI $)$ 
a)

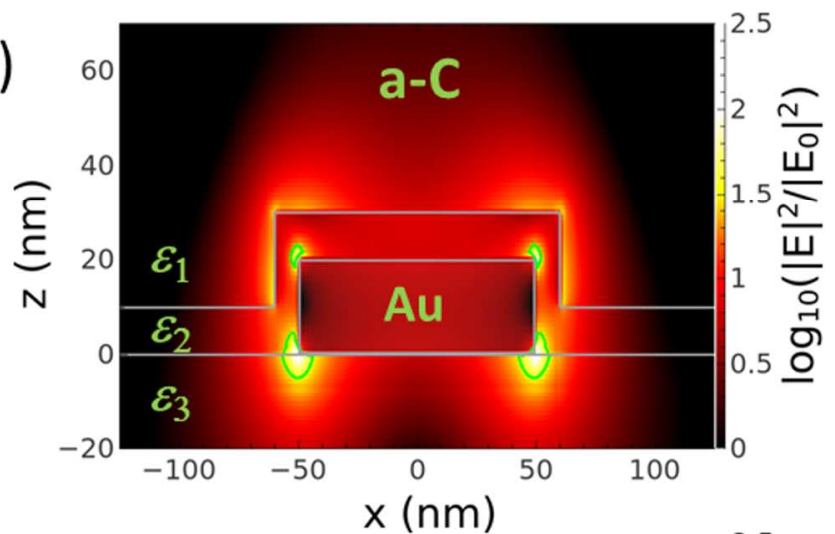

b)

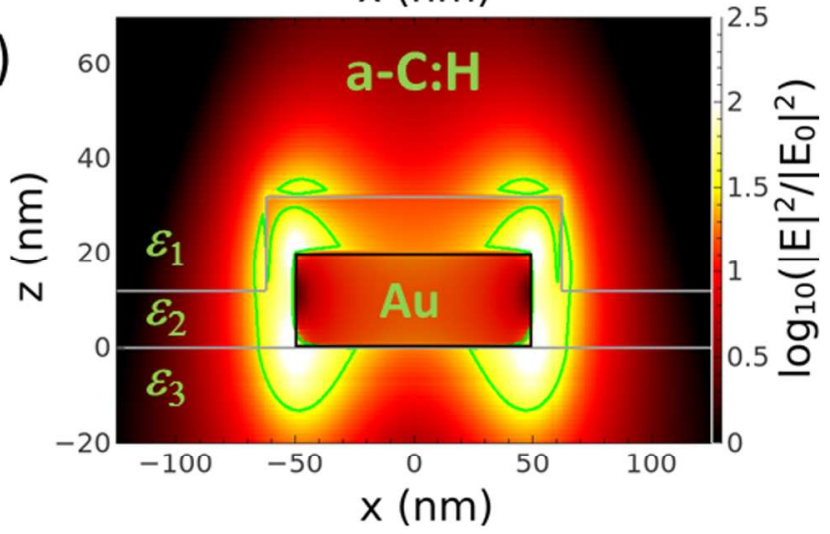

c)

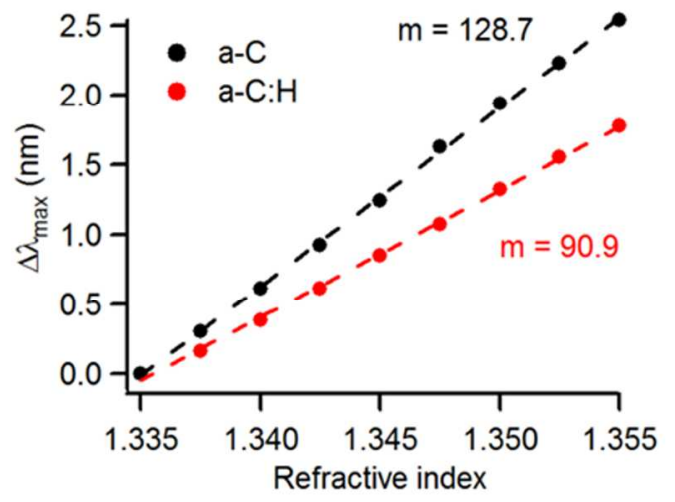

Figure 2. Electric field intensity distribution around isolated nanodisks immersed in PBS obtained via FDTD modelling at the wavelength corresponding to the maximum of the LSPR. The refractive indeces used in the simulation are reported as $\varepsilon_{1}, \varepsilon_{2}$ and $\varepsilon_{3}$ for the aqueous medium $\left(\varepsilon_{1}=1.333\right)$, the carbon coating and the glass substrate, respectively. The green line in the graphs indicates a factor of 30 increase in the electric field intensity. (a) Field distribution around an isolated $\mathrm{Au} / \mathrm{a}-\mathrm{C}$ coated nanodisk at $797 \mathrm{~nm}$; (b) Field distribution around an isolated Au/a-C:H coated nanodisk at $748 \mathrm{~nm}$. (c) Calibration plots obtained via FDTD methods for $\mathrm{Au} / \mathrm{a}-\mathrm{C}$ (black line) and $\mathrm{Au} / \mathrm{a}-\mathrm{C}: \mathrm{H}$ (red line) coated nanodisks; the slope yielding the analytical sensitivity is reported next to the corresponding curve.

$$
80 \times 139 \mathrm{~mm}(300 \times 300 \text { DPI })
$$


a)

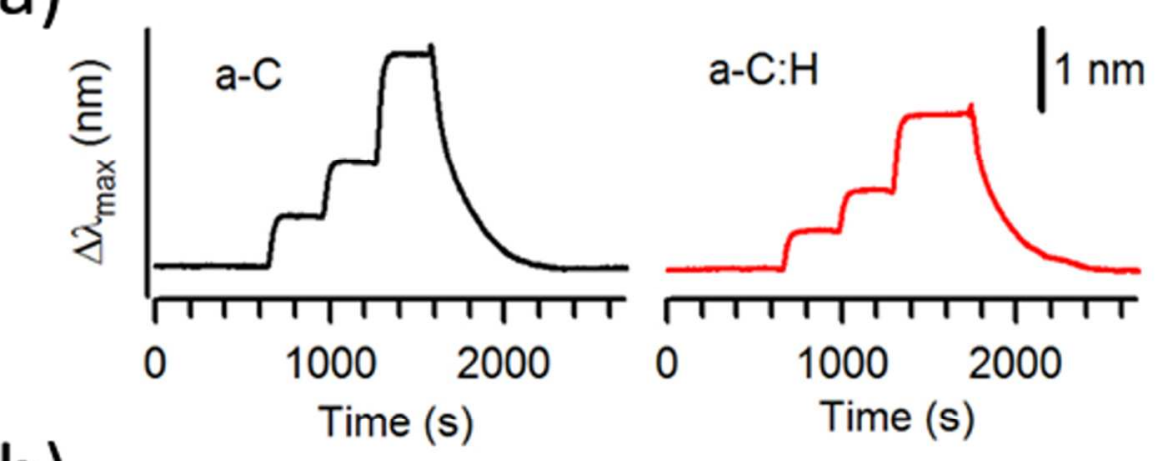

b)

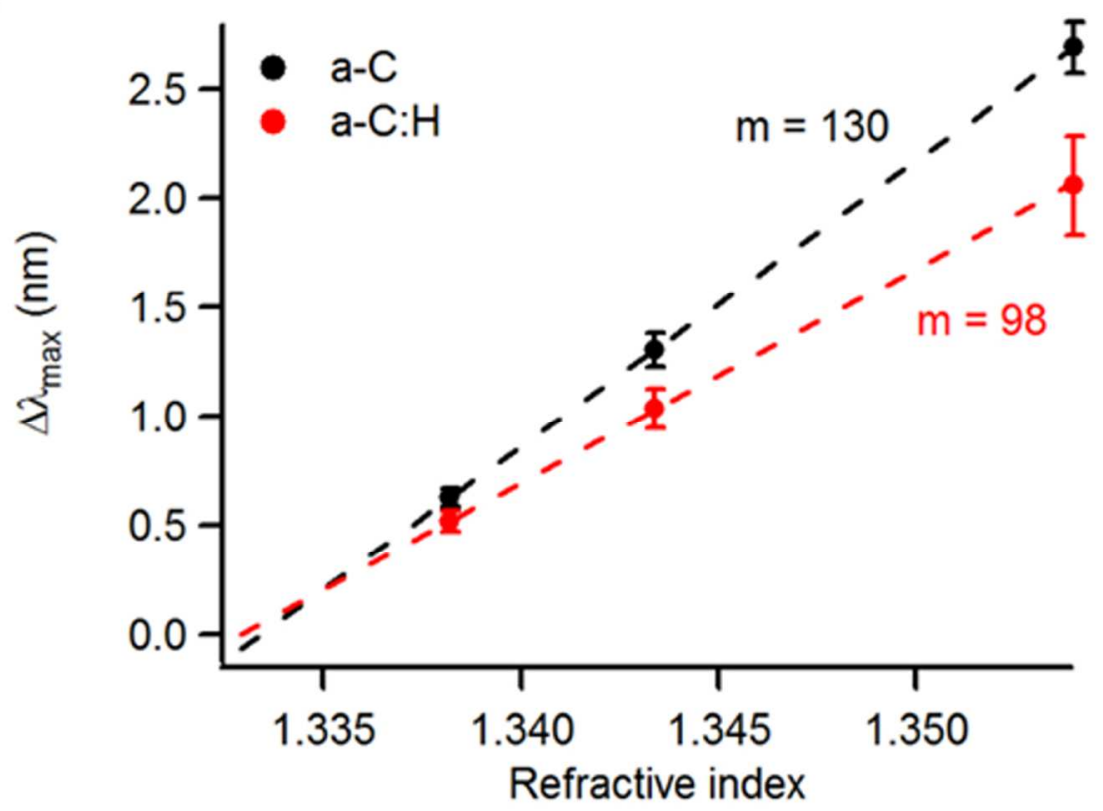

Figure 3. Sensitivity test obtained at a-C (black, left) and a-C:H (red, right) coated sensors. (a) NPS shift $\Delta \lambda_{\max }$ as a function of time measured after water/ethylene glycol solutions of different refractive index are injected into the cell. (b) Calibration plot of measured $\Delta \lambda \max$ vs. refractive index of the water/ethylene glycol solution; the slope yielding the analytical sensitivity is reported next to the corresponding curve. Error bars indicate $95 \%$ C.I. calculated from sample size $n=5$ and 3 for a-C and a-C:H, respectively.

$80 \times 87 \mathrm{~mm}(300 \times 300$ DPI $)$ 
1

2

3

4

5

6

7

8

9

10

11

12

13

14

15

16

17

18

19

20

21

22

23

24

25

26

27

28

29

30

31

32

33

34

35

36

37

38

39

40

41

42

43

44

45

46

47

48

49

50

51

52

53

54

55

56

57

58

59

60
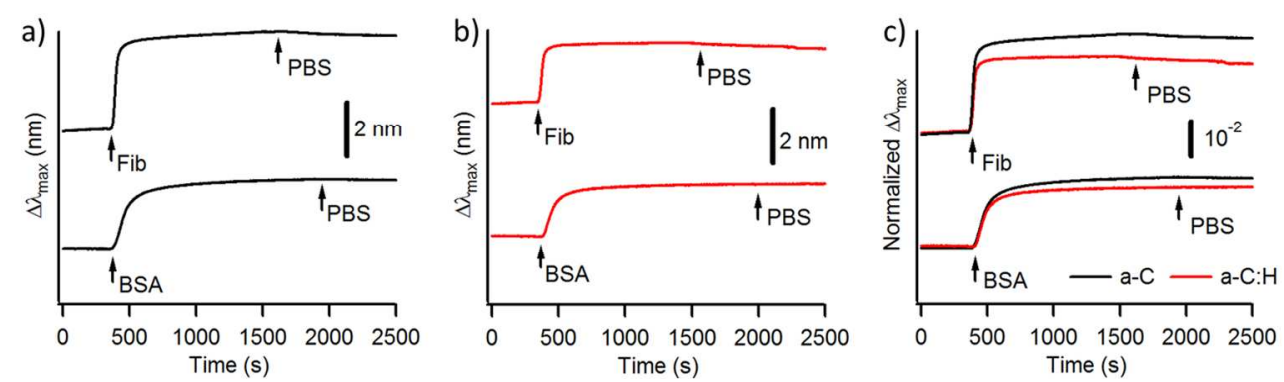

Figure 4. NPS wavelength shift, $\Delta \lambda_{\max }$, as a function of time, measured at (a) a-C and (b) a-C:H coated sensors for in situ protein experiments. (c) Normalized $\Delta \lambda_{\max }$ as a function of time calculated using the initial calibration of the sensor at both a-C (black line) and $\mathrm{a}-\mathrm{C}: \mathrm{H}$ (red line) surfaces. The arrows indicate the time of the injection of BSA, Fib and PBS solutions into the flow cell.

$160 \times 46 \mathrm{~mm}(300 \times 300 \mathrm{DPI})$ 


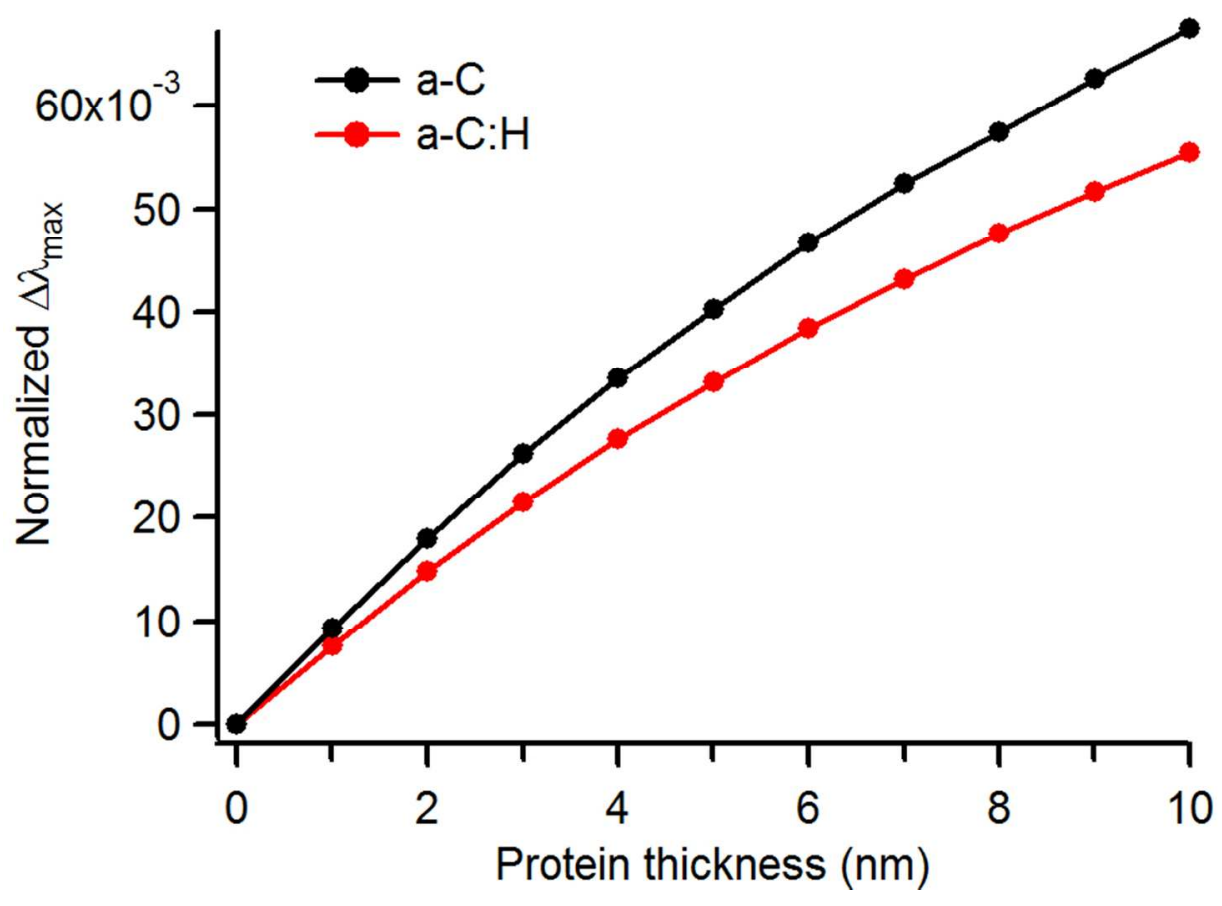

Figure 5. Simulated normalized $\Delta \lambda_{\max }$ for a-C (black) and a-C:H (red) coated sensors calculated for various thicknesses of the protein layer using the FDTD method.

$81 \times 57 \mathrm{~mm}(300 \times 300 \mathrm{DPI})$ 


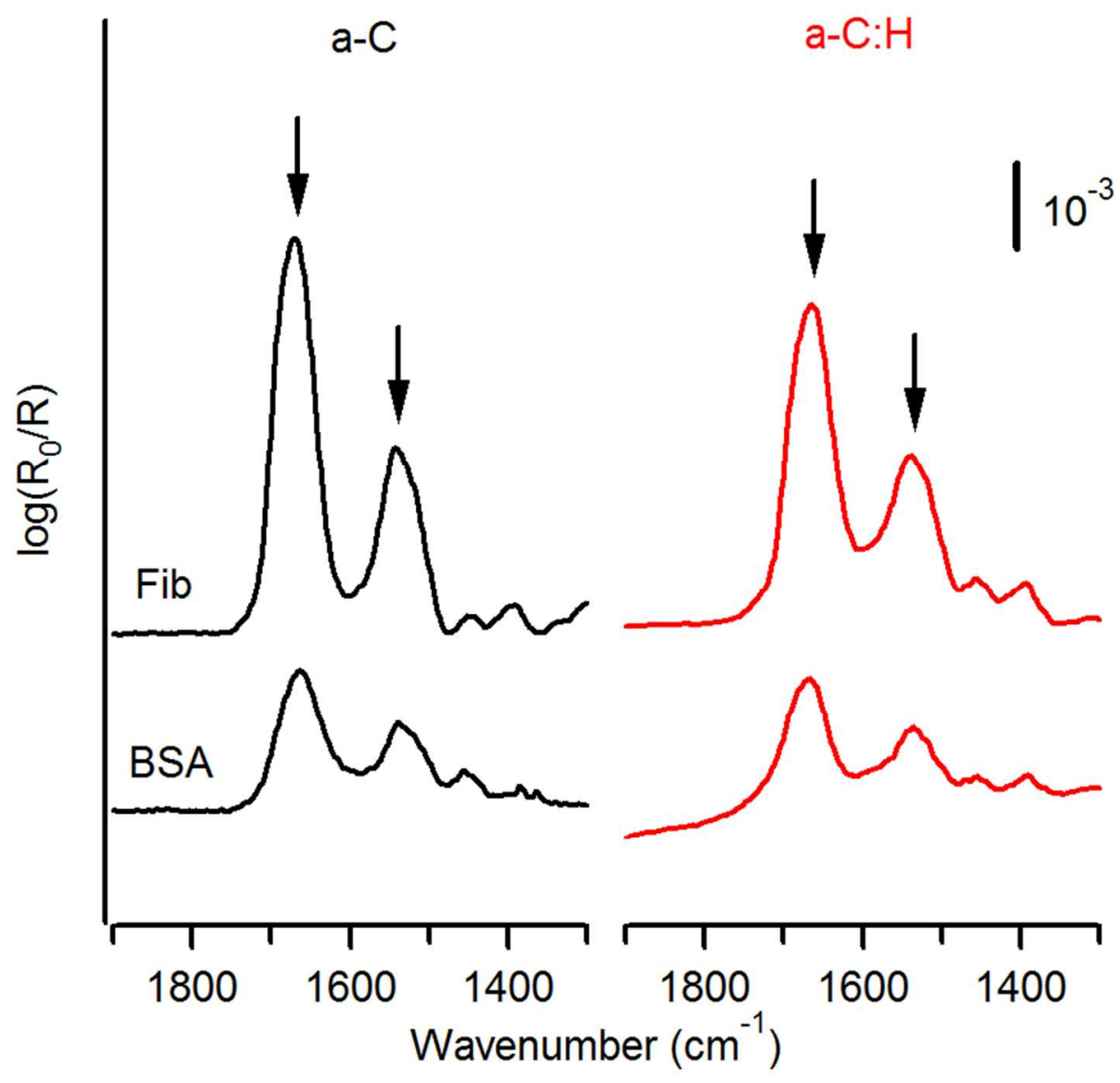

Figure 6. IRRAS spectra of a-C (black, left) and a-C:H (red, right) substrates after $1 \mathrm{~h}$ incubation with BSA (top) and Fib (bottom) solutions. The arrows indicate the peak positions of the amide I and amide II bands. Spectra were baseline corrected and $\mathrm{a}-\mathrm{C}: \mathrm{H}$ peaks were corrected for optical enhancement, as indicated in the experimental section, to facilitate comparison of peak intensities.

$79 \times 73 \mathrm{~mm}(300 \times 300 \mathrm{DPI})$ 
a)

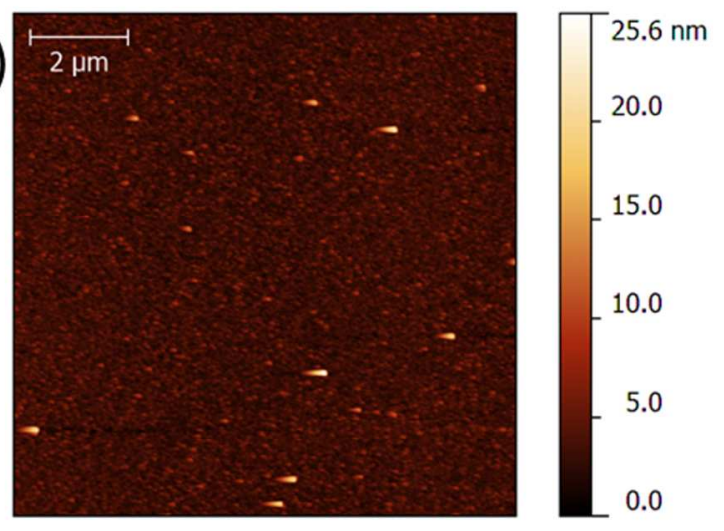

b)

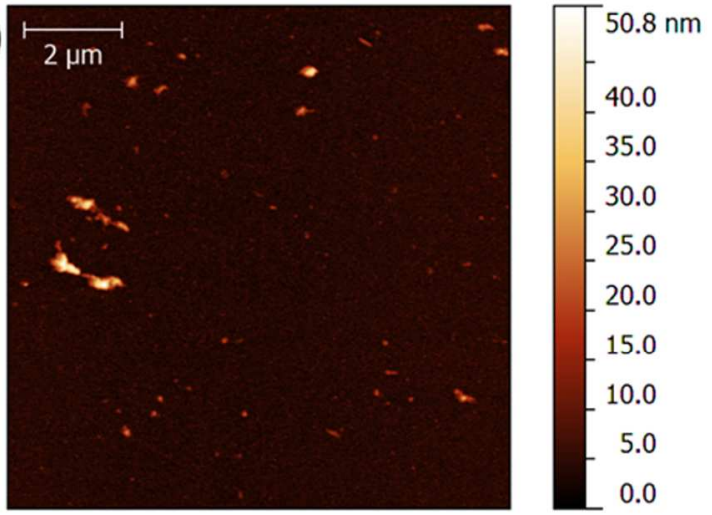

c)
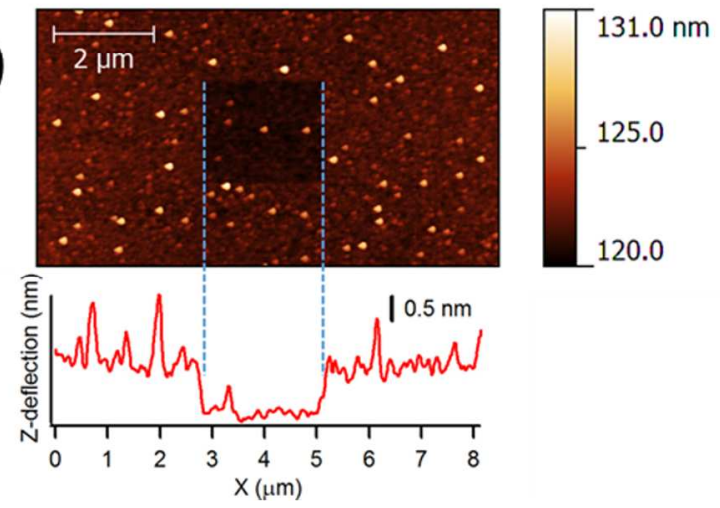

Figure 7. AFM topographic images of a-C:H surfaces after incubation with (a) BSA and (b) Fib solutions; thickness of (c) BSA layer adsorbed at an a-C surface.

$80 \times 155 \mathrm{~mm}(300 \times 300 \mathrm{DPI})$ 